INTERNATIONAL DESIGN CONFERENCE - DESIGN 2018

https://doi.org/10.21278/idc.2018.0401

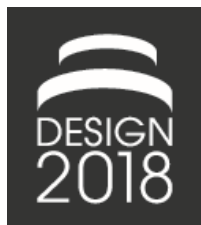

\title{
MODELLING CHANGE WITH AN INTEGRATED APPROACH TO MANUFACTURING SYSTEM DESIGN
}

\author{
H. Olmez, M. Hassannezhad, N. Ball and P. J. Clarkson
}

\begin{abstract}
This paper proposes a model that integrates information from product, process and organisation domains with a view to help manage these complex interrelationships with multiple layers of interaction. This model incorporates an integrated mechanism that simulates change effects during the design of complex manufacturing system by populating a Multi-layered Domain Matrix (MDM) and applying a Change Prediction Model (CPM) propagation mechanism to interconnected elements.
\end{abstract}

Keywords: complex systems, change prediction, design structure matrix (DSM), resilient design, manufacturing system

\section{Introduction}

The competitive manufacturing environment forces manufacturers to respond rapidly and in a costefficient manner to their change requirements. Manufacturing systems need to be robust and adaptable to their facilities, technologies, methods, people, and products against continuous changes. Improving the performance of manufacturing systems by reducing their complexity offers a major competitive advantage for an organisation to satisfy customer expectation effectively.

This paper presents a model that attempts to support this process by integrating system domains (product, process, and organisation) to decrease the number of interfaces and simulating the propagation of the types of change that a manufacturing system may face. This paper presents a multilayer network model of change propagation and investigates a case study of a real-world engineering project. The model provides a roadmap with which to evaluate existing products and manufacturing processes in a systemic way to improve manufacturing system design. The proposed approach involves the use of simulation and modelling tools in a design environment, which allows a diverse group of researchers, manufacturers, and suppliers to work within a comprehensive network of shared knowledge.

\section{Background}

\subsection{Manufacturing System Design}

Manufacturing systems operate in a constantly changing environment (Wiendahl and Heger, 2005). Pressures from globalisation have forced manufacturing enterprises to respond rapidly to changes such as the constant innovation of product, technology, or requirements from customer demands, reducing product cycle, and cost (Nylund et al., 2009). In order to achieve these changes: a manufacturing system that is designed strategically and integrated properly with the rest of the 
manufacturing functions plays an important role (Vaughn et al., 2002). Manufacturers need to understand how design issues affect to interact with various components of a manufacturing system in order to make a strategic decision. Evaluation of Manufacturing System Design (MSD) impacts and the effective communication of those impacts across the MSD domains need an integrated approach (Cochran et al., 2001; Kim, 2004).

The design of manufacturing systems to support a company's business strategy is a difficult challenge in terms of complexity because of the high level of connectivity between elements and subsystems challenging problem because the time, cost, and resources that need to be allocated to effect the change are dependent on its potential impact (Eckert et al., 2004; Wickel and Lindemann, 2015). The relationship and dependencies between change requirements and system components are fundamental and modelling information needs to be considered to fully describe the propagation of engineering changes. Such information can aid modelling requirements to support the development of change prediction methods (Koh et al., 2012).

The effectiveness and efficiency of the manufacturing systems design could be greatly increased if an integrated perspective to system domains and activities could be provided (Nylund et al., 2009). The intention of this research is to integrate the core domains - i.e. product, process, and organisation - into one system by the efficient use of existing skills and knowledge to examine change propagation and reduce the complexity of the system design (Nylund et al., 2009). A systematic representation of MSD can help manufacturing engineers and designers to capture and examine changes in the interrelationships among the different elements of a system design for decision-making. The following section, therefore, provides theoretical explanations of modelling change within MSD. A systematic way is then presented to examine the interactions among manufacturing system domains with design perspective in to understand change impact and change management.

\subsection{Modelling change in Manufacturing System Design}

Change is one of the most powerful driving factors of design because changes provide a significant challenge in a system management. A change can either interrupt a current task or delay it until a resource becomes available. Changes can also generate additional connectivity between products, thus increasing the complexity of the organisation (Eckert et al., 2005). The modification of a single subsystem can dramatically turn into an expensive redesign that requires adaptations to a wide range of components (Jarratt et al., 2002).

Hence, a company's ability to undertake and manage change can be influenced greatly by their understanding of the links that exist between different parts of the product and the impact that these will have on the propagation of change. The accurate prediction of this change propagation provides a significant challenge in the management of redesign and customization (Clarkson et al., 2001). Therefore, it is important to identify the need for change early in the design process, since the later a change or the impact of a change is detected the more expensive it becomes to process (Clarkson et al., 2001). An accurate impact assessment of a design change, in terms of time, cost and resource, is essential when deciding whether to implement a change and where applicable, determining the cost to be charged for any rework. In order to evaluate effects of change on products, which consist of several thousand components, it is important that the product is decomposed into understandable and manageable representations of the system (Ariyo et al., 2007).

Manufacturers need a systematic way to define changes because many manufacturing industries are subject to high levels of change and considerable amounts of time and cost required to make changeovers (Cahlarek and Jin, 2004). Making a change to a product, process or organisation within manufacturing system is a manageable process in most cases. Nylund et al. (2009) present a model of integrated manufacturing systems, which consists of manufacturing entities of products, resources, and orders, which have different roles in the manufacturing system. The entities are connected through the process, production, and business domains. Eger et al. (2005) believe the significant cause of the problem in managing change originates from a lack of understanding of the connectivity between products and process in industry and they observe the impact of changes at levels of Product, Process, Organisation, and External factors. Myklebust (2002) similarly divides a manufacturing system and service design into three key domains: Product domain, Process domain (A manufacturing process), 
Resource domain (organisation). These three dimensions are sufficient for the analysis of design processes that integrate manufacturing systems and services (Myklebust, 2002; Haq et al., 2011; Vasantha et al., 2012).

One significant cause of the problem in managing change originates from a lack of understanding of the connectivity between products and processes in industry. The source of change, interdependencies between parts and systems, types of propagation behaviour, consequences of change on product quality, cost, and time to market, and the state of tolerance margins on key parameters need to be taken into consideration for successful change management (Masmoudi et al., 2017). A characterisation of change impacts using such methods needs the prediction of the possible consequences of system changes in its structure and performance (Reddi and Moon, 2009).

The desire to capture and manage changes and change propagation within manufacturing systems requires an integrated model. (Ahmad et al., 2013). An integrated environment, connecting the manufacturing activities, can be one of the main enablers for successful operation in the global markets. The integration of (a) design and development activities and (b) products and production systems into one system enables existing skills and knowledge to be used more efficiently (Nylund et al., 2009). Therefore, the following section reviews the integrated models of changes to increase the understanding of the representation of a manufacturing system to manage changes systematically.

\subsection{Integrated modelling of changes in Manufacturing System Design}

An integrative change model of manufacturing systems incorporates a wide range of knowledge and information for decision making in risk assessment of change effects. This paper proposes an approach to integrate design tasks within a manufacturing system, which explains how to analyse and identify the type of dependencies that can exist between two or more domains in a system. The dependencies between the parameters of structural components are identified through the knowledge of designers. These dependencies can be characterized qualitatively (Cohen et al., 2000; Furtmeier and Tormmelien, 2010) and/or quantitatively (Clarkson et al., 2004; Hamraz et al., 2013). A dependency analysis method or an effective change propagation analysis method requires characterising the relationship between parts and domains and translating it into a system. Analysing the relations and patterns of the changes through the network of dependencies requires a systematic dependency analysis and multiple levels of analysis allow greater insight into linkages that increase the system understanding.

Matrix-based representations can increase the system understanding by presenting a holistic view of connectivity. The most well known the matrix-based change model is the contribution from Clarkson et al. (2001). Initially, Clarkson et al. (2001) developed a Change Propagation Method by using numeric (Design structure Matrices) DSMs. Clarkson et al. (2001) built the DSM according to the parametric relationships between design components. The DSM is a matrix-based representation for modelling the elements of a system and their interconnections (Browning, 2001; Eppinger and Browning, 2012). A DSM decomposes a product into its key elements (which may include components, subsystems and parameters) and defines the ways in which change may propagate between them. These models are normally generated in interviews with experts, which result in estimates of the propagation of change at an element-level (Wickel and Lindemann, 2015).

The challenge is to map the process accurately in a DSM because dependencies are difficult to capture, and the DSM cannot be directly defined in an exact state. The MDM extends the capabilities of the DSM by integrating multiple domains and enabling the deduction of indirect dependencies (Furtmeier and Tormmelien, 2010) within domains and across domain boundaries. In this paper, an approach towards a matrix-based system model is presented which applies a systematic process to the modelling of a whole system. The resulting multi-level system elements and the hierarchical system decomposition can be used to simulate manufacturing system property changes and their propagation throughout the system.

Although the DSM provides no direct indication as to the likelihood or redesign, it may be used as the basis for a process simulation that includes consideration of rework (Giffin et al., 2009). Through the identification of critical process structures that impact cost and schedule risk, the method supports the analysis of the impact of planned design changes. This predictive model calculates a combined risk of 
propagation from its direct and indirect components connections. Eckert et al. (2004) examined this change propagation process through the case study in the aerospace industry and identified two types of changes: emergent changes and initiated changes. The study was conducted with the company employees based on the interviews to capture the design knowledge and experience. Further, this work, Clarkson et al. (2004) developed a computer tool to identify the risk of a change.

This paper aims to identify the research gap in the most relevant research studies (in engineering design), which are shown the comparison between the studies in Table 1. This comparison considers two characteristics - the underlying model and the types of knowledge represented in that model. The referenced works use Multi-Domain Matrix (MDM) as an integrated model to identify the indirect dependencies. However, the system analysis approaches are different in the first two references. Eichenger et al. (2006) analysed their system with AID Process Optimisation Process in Component, Functions, and Parameter Layers during a matrix filling process. This analysis demonstrated that indirect dependencies could be made clear to the team members more easily. However, to prove the assumption that many indirect relations are likely to cause also a direct relation between elements, Pasqual and de Weck (2011) applied Engineer-CPI (Engineering Change Propagation Index) and Propagation Directness (PD) only in social, change and product layers and that approach appeared to create extra workload for engineers.

The last three references are from the Cambridge Engineering Design Centre (EDC). Koh et al. (2011), Hamraz et al. (2012), Hassannezhad et al. (2017) used MDM and CPM in different domains with different analysis approaches. Koh et al. (2011) used a matrix-based approach, which built on the HoQ (House of Quality) and the CPM methods to model the performance of different change options during the design and development of complex products. The objective of this research was to highlight how much each change option could affect the product requirements by considering potential change propagation. Hamraz et al. (2012) proposed the function-behaviour-structure (FBS) linkage model, a multi-domain model that combines concepts of both the function-behaviour-structure model with the change prediction method (CPM). Hassannezhad et al. (2017) combined the concepts of CPM and Systems Dynamics to address complexity of large mature organisations through identifying the key elements (in terms of their impact in propagating changes) and understanding their dynamic behaviour. The authors developed a multi-domain CPM considering the strategic, tactical, and operational levels of decision-making, from the views of the organisation, employee, and customer.

Table 1. Related works in modelling change with an integrated approach

\begin{tabular}{lll}
\hline Reference & The methodology & Types of Knowledge (layers) \\
\hline Eichinger et al. (2006) & $\begin{array}{l}\text { MDM-AID (Analysing Indirect } \\
\text { Dependencies) Optimization Process }\end{array}$ & $\begin{array}{l}\text { Component, Functions, and } \\
\text { Parameter Layers }\end{array}$ \\
Pasqual and de Weck (2011) & $\begin{array}{l}\text { MDM, CPI (Change Propagation } \\
\text { Index), and Propagation Directness } \\
\text { (PD) }\end{array}$ & $\begin{array}{l}\text { Social, Change, and Product } \\
\text { Layers }\end{array}$ \\
Koh et al. (2012) & $\begin{array}{l}\text { MDM, CPM, and House of Quality } \\
\text { (HoQ) }\end{array}$ & $\begin{array}{l}\text { Product Components, Change } \\
\text { Options, and Product }\end{array}$ \\
& MDM and CPM (integration of & $\begin{array}{l}\text { Requirements Layers } \\
\text { Function, Behaviour, and Structure } \\
\text { Hamraz et al. (2012) }\end{array}$ \\
CPM with FBS ontology) & $\begin{array}{l}\text { layers } \\
\text { Strategic, Tactical, and } \\
\text { Hassannezhad et al. (2017) }\end{array}$ \\
& OpMerational layers of decision- \\
& & $\begin{array}{l}\text { making; and from the views of } \\
\text { organisation, employee, and } \\
\text { customer }\end{array}$ \\
\hline
\end{tabular}


This paper develops a new network-based analysis technique and applies a number of existing change propagation analysis methods to a data set from the manufacturing industry.

\subsection{The research gap}

There are several challenges that need to be overcome to map and integrate product, process, and organisation perspectives:

1. The scope and focus of integrated models need to be clearly identified;

2. A creation and maintenance strategy for integrated models needs to be defined;

3. Visualisation techniques and tools need to be developed to help designers work with the large volume of information that will be generated by the change propagation processes operating on integrated models.

Changing product requirements are one of the main reasons for manufacturing system changes. Such changes can affect any number of people and can take any length of time and occur when change requests are made to products, components, operation techniques, processes, supplies, or regulations. Most research studies into change propagation have focussed on change management in engineering design, product development, and complexity (Wright, 1997); the effect of changing requirements has not been central to many research studies.

A Multiple Domain Matrices (MDM) have been used to model the connections between different process, product and organisational domains and these techniques can be extended to identify the engineering change and rework arising from a changed requirement providing that requirements can be directly mapped to the related components and tasks. Therefore this paper extends the existing models based on Product, Process and Organisational layers by adding a Requirements layer and provides a comparison between the resulting interconnection and integration connectivity of this four-layer model with those listed in Table 1.

\section{The proposed approach}

In this section, a modelling approach is presented to support the analysis and evaluation of change requirements in an existing assembly design process. The approach describes how an existing system could be analysed and deconstructed to develop an integrated MDM model from which change propagations can be simulated and evaluated using the Change Prediction Method (CPM).

\subsection{Overview of the proposed approach}

The approach proposed in this paper is based on the analysis of a system change model that is represented by a DSM in terms of product, process, and organisation elements. The Change Prediction Method (CPM) proposed by Clarkson et al. (2004) is a numerical method, which uses a DSM model of dependencies between components to visualise the overall risk of change being propagated to other components when one component is changed. The CPM is a matrix, where the column elements indicate components that initiate changes; the row elements indicate components that receive changes and the cells between two given components values.

A CPM analyses system components according to their change propagation as defined by likelihood, impact, and risk parameters. Experts produce the values for the likelihood and risk e propagation parameters; the value for the risk parameter is then computed based on component connectivity captured in the DSM. The approach proposed in this research defines a procedure for $s$ the creation of CPM matrices based on five stages as follows:

Stage 1: Decompose the case study system into its elements and create a CPM matrix from these elements. Before a system can be analysed it must first be broken down into sub-systems, allowing the system to be viewed as a collection of parts whose designs can affect one another. A designer's experience with the original design can help to elicit how a system may be broken down into an appropriate number of sub-systems. Identifying the right level of detail in developing a model is critical; a model with fewer than 50 components is recommended (Clarkson et al., 2001);

Stage 2: Identify the direct dependencies between the system elements and create connections within the CPM matrix to mirror these dependencies; 
Stage 3: Compute the predictive matrix by populating each connection with an estimate for the likelihood and impact of changes between the connected elements; Note that such estimates are not bidirectional i.e. the estimate for A to B may not be the same for B to A. The resulting matrix represents the direct risk of change propagating between linked sub-systems; indirect change propagation requires the involvement of at least one intermediate sub-system and this forms a chain of change propagation. The combined impact of changing one component on another is the sum of the direct and indirect effects;

Stage 4: Compute the combined change propagation by applying the CPM algorithm for a specific number of steps. In this stage, it is important to understand what elements of the system are subject to direct changes and how such changes can propagate to impact elements that have no direct dependencies. The CPM toolbox of CAM is used to analyse how the level of compound risk is correlated with both the level of element interconnectivity and to the likelihood and impact assigned to direct connections between elements;

Stage 5: Use the model to support decision making by identifying which elements could have the biggest impact if changed and/or which elements are most likely to be impacted by changes to any other element. The model can be used to assist in the decision-making process by reviewing the effect of the change on each domain in terms of the total combined risk of elements and combined risk variation on different likelihood outcomes. The CAM modeller can be used to identify the riskiest elements (that are most likely to initiate problems) and those elements that are most sensitive to issues that arise as a result. The analysis results of such analyses will vary depending on the number of change propagation steps that are applied.

\subsection{Case study}

This paper demonstrates the application of the approach on a case study of a Kitchen Assembly System that is a module in a pre-fabricated modular building. The complexity of the assembly process of modular buildings is determined by whether they contain a kitchen, a bathroom, a utility cupboard, or a combination of all three. Buildings that contain a kitchen module require extra work on the finishing line to install the required units and appliances, and to make the required electrical and plumbing connections. This is due to the kitchen module being one of the governing factors in the complexity of a modular assembly, and its potential to act as a bottleneck in the assembly process. Therefore, dealing with any possible changes (e.g., kitchen style, kitchen layout, supplier, etc.) in the kitchen, assembly process will lead to improvements in the overall module assembly.

The company involved in this case study is looking for ways to improve the resilience of novel construction elements and their associated design and manufacturing processes. Fundamental to this requirement is the importance of project management decision making the cross team and crosscompany information flow and tools necessary to support effective decisions particularly focussing on the impact of different supplier specifications on the assembly of the kitchen module. It was clear from interviews conducted with a supply chain manager at the construction company, that change propagation is unwanted and that there is a need to understand its effects on the system. Company employees agreed that there was a need for a tool to assess change propagation effects at an early stage in the design process.

Based on several discussions with process engineers from the company, 4 domains and 18 components were identified to represent the entire Kitchen Assembly System. The identification criteria were a balance between a manageable number of components and the right level of detail for meaningful analysis. The identification of linkages between components and the assessment of their change propagation likelihood and impact is the next step resulted in 87 direct linkages between the 18 components held in the DSM. Each direct linkage between two components could be made up of more than one linkage type and the process engineers provided an indication of the likelihood and impact of change on each link as they analysed each connection. The scale used was 'Low', 'Medium', and 'High'. Information such as redesign cost and lead-time was also produced using the scale of 0 (Low) to 1 (High).

The proposed approach (combining the Multi-Domain Matrix and Change Prediction Method models) has been used to characterize changes (requested by internal or external customers) in terms of their 
impact on the assembly time of a module. This characterization of change type will enable the manufacturer to reduce the "takt" time (an average time to produce one finished product) on their main assembly line, and increase the volume of modular buildings that can be produced. The case study demonstrates the application of the MDM as both a process-mapping tool and a decision support tool that can improve the understanding of the product-process relationship in the design of a kitchen module.

\subsection{Model application and the results}

The data were analysed by the Change Prediction Method (CPM) tool developed in the Cambridge Engineering Design Centre. An implementation of the method behind stages 2, 3 and 4 are freely available in the software program Cambridge Advanced Modeller (CAM) in Cambridge EDC website (Wynn et al., 2012). The CPM tool analyses direct and indirect change propagation between components and is capable to classify components according to their change characteristics. To proceed with the analysis, the 'Low', 'Medium', and 'High' scale used for propagation change likelihood and the impact was converted to numerical values of $0.3 ; 0.5 ; 0.8$. By classifying the components according to their change propagation characteristics, different rankings and plots could be generated for each analysis. The application of the approach and generation of the simulation results are presented in Figure 1.

Stage 1: The specific model proposed here is composed of three primary layers: namely, the product layer, process layer, and organisational layer with an additional requirement layer also incorporated into this model as a secondary layer. Each layer is associated with a particular aspect of the process as shown in Figure 1 (1b-1c);

Stage 2: In this stage, shown in Figure 1.2, the dependencies between the elements and between domains are identified in the case study and mapped and onto each DSM within the MDM. Once the "intradomain" connections have been established (2a) then the "inter-domain" connections must be identified and mapped onto DSMs between domains $(2 b)$;

Stage 3: This stage (Figure 1.3) establishes a detailed understanding of relationships between elements. Each relationship is characterized by two values - the likelihood (frequency) of change and its predicted impact (high, medium, low). Once this information has been extracted from the case study, it is transferred into the MDM using DSM toolbox of the CAM system so that it can be applied in the analysis of impacts and risks in the next stage.

Stage 4: As the result of applying CPM algorithm in the case study, the indirect connections between the 18 elements can be generated using a three-step propagation analysis as shown in Figure 1(4a). These connections can be mapped onto a compound risk plot (Figure 1(4b)), which enables the user to simultaneously change the characteristics of the direct relationships between elements and evaluate the impact of these changes on the indirect relationships that result from change propagation through the MDM. The components that fall within the top-left region of the Risk Plot (Figure 1(4b)) have a low likelihood of change propagation but will incur a high amount of redesign effort if a change is required.

Therefore, components that fall within this region should be standardised. If a change is required, the connectivity between these components and the rest of the components should be reduced to further decrease the likelihood of changes in propagation. Components that fall within the bottom-right region of the risk plot (Figure 1(4b)) have a high likelihood of change propagation but require a low amount of effort if a change is required. Therefore, if a change is required, these components can be redesigned as flexible components. This is to reduce the impact of future changes, as these components are very likely to be changed. Components that fall within the bottom-left region have low likelihood and impact of change propagation. These are the least critical components and platform strategies are optional. 


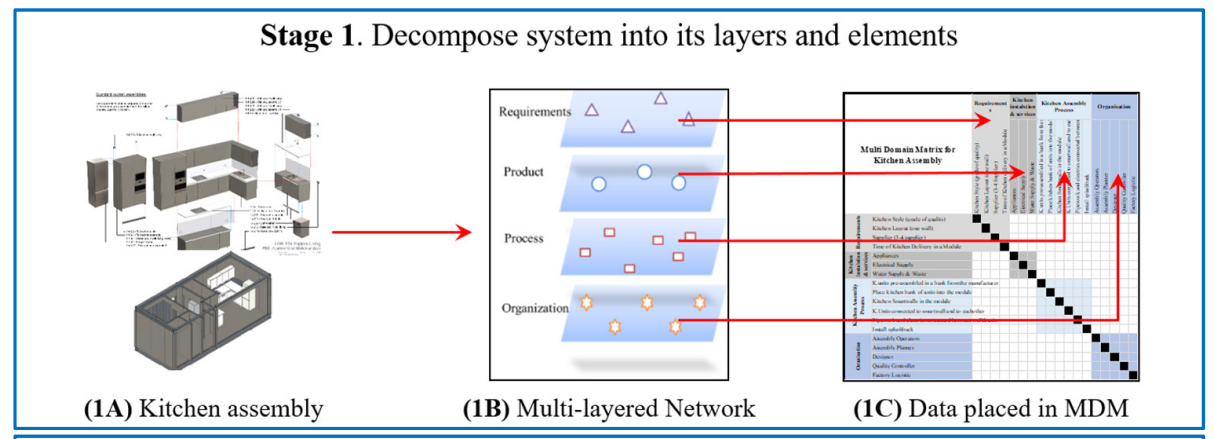

Stage 2. Capture dependencies between the layers and elements

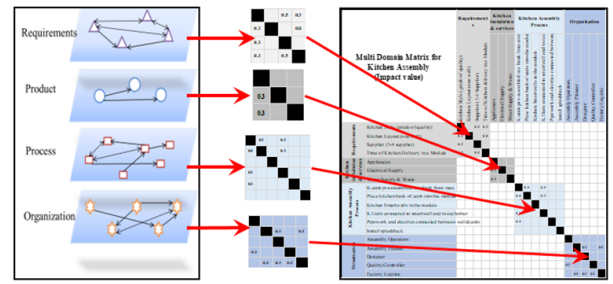

(2A) Interconnected Network Relationships

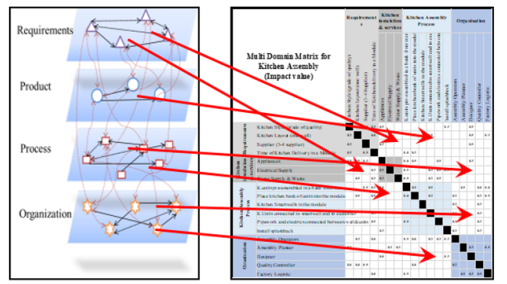

(2B) Integrated Network Relationships

Stage 3. Compute Predictive Matrix

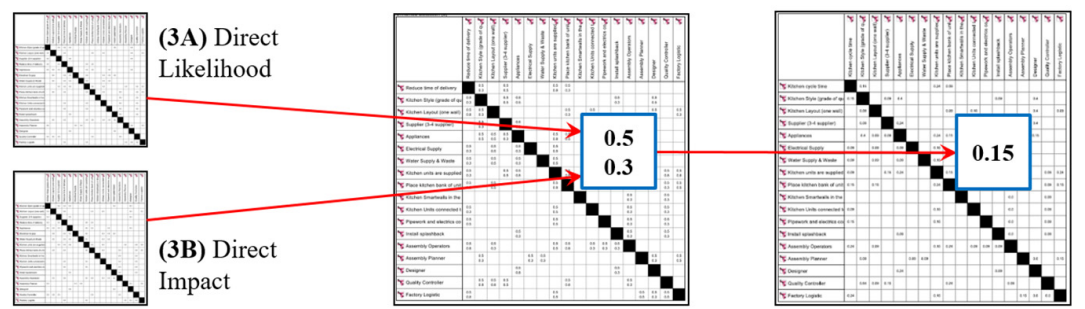

(3C) Connected Likelihood and Impact Values

(3D) Direct Risk

Stage 4. Compute combined change propagation

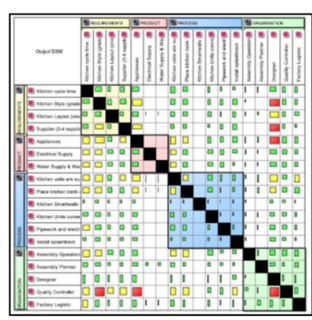

(4A) Apply CPM algorithm

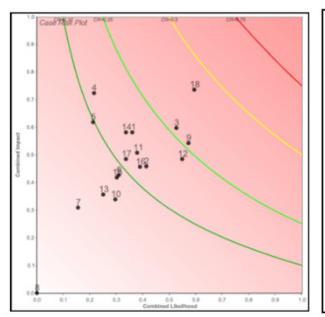

(4B) Generate Combined Risk plot

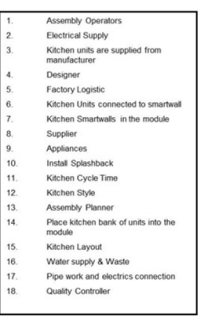

Stage 5. Use the model to support decision making

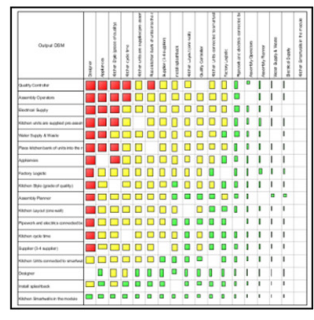

(5A) Kitchen Assembly System Risk Matrix (Reordered)

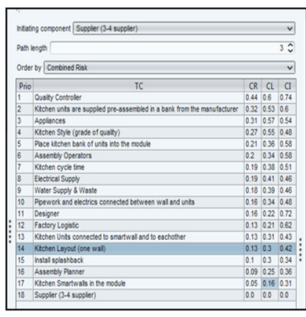

(5B) Prioritised change risk list for Suppliers

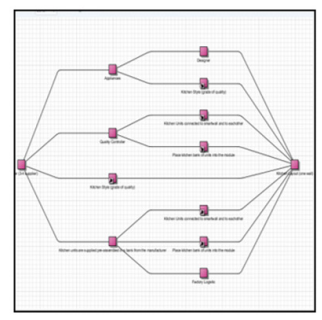

(5C) Propagation path from Suppliers to Kitchen Layout

Figure 1. Application of the change modelling method in the Kitchen Assembly case 
On the other hand, components, which fall within the top-right region, are likely to be changed and would require high redesign effort. In an ideal case, no component should fall within this region. Recommendations on the appropriate design strategies can be made for these components by extending the analysis to take into account their outgoing change risk.

Stage 5: At this stage, Figure 1.5, the likelihood of change for each system component can be visualized as a one-axis scatter plot. In this plot, any elements that is placed above or to the right of another system component has relatively higher change impact or likelihood, and is therefore more susceptible to change.. System elements with high axis plot have a high impact on other system elements and so should be made more resistant to change in order to avoid propagating further changes to others. System elements with low axis do not affect other system elements as much and therefore it will be easier to accommodate future change. Overall, the proposed approach reflected an acceptable outcome in addressing in change propagation prediction in the kitchen assembly system. Providing reliable MDMs (avoiding unnecessary connectivity) data and the collected data associated with likelihoods and impacts in CPM can affect the performance of the model. The following section discusses how the model to be improved.

\subsection{Discussion and further analysis}

The CPM approach presented in this paper enables a designer to explore a scenario for how changes on "Supplier" element can affect the other elements and overall system performance. The key relationships in a large system can often span multiple levels across different domains.

It can be seen from the Figure 2 "combined impact (CI) versus combined likelihood (CL)" chart those system elements such as "Kitchen Smartwall in the module (7), "Install Splashback(10)" and "Assembly Planner (13)" have low CI and CL, suggesting that this element has less change impact compare with the rest of system elements. Therefore, this could suggest that the elements 7, 10 and 13 are suitable for standardization. On the other hand, "Designer (4)" and "Factory Logistic (5) have high $\mathrm{CI}$ and low CL value which suggests that although they are not very likely to change. If the change is required it should be considered with caution.

Approaches to reduce the impact of changing the Designer and Factory Logistic can also be considered; however, the benefit doing this would be minimal as the likelihood of change is quite small. Possible suggestions for system components with moderate CI and CL are less clear; for example; "Kitchen Layout (16)" and "Electrical Supply (2) are in the centre of the chart with moderate impact and the likelihood of change and therefore they are less critical. In order to reduce their change likelihood or impact, it is better to look at the one-axis scatter plot (Koh et al., 2013). In this chart, Kitchen Layout (16) and Electrical Supply (2) have low values (less than 0.5 out of a $0-1$ range which implies that they will have a low impact on the other parts of the Kitchen Assembly System and hence could be considered for a change in the future.

The system elements in the upper right quadrant of the risk plot (Figure 1(4b)) such as "Quality Controller (18) and "Kitchen units are supplied from the manufacturer (3)" are most likely to be changed and have high change impact if a change is required. Therefore, these elements should be subject to detailed analysis via the one-axis scatter plot. It can be seen that the "Kitchen units are supplied from the manufacturer (3)"'has a moderately low value in the plot which implies that it has a low influence on other system components if changed and is more able to absorb any future changes. In contrast, the "Quality Controller (18) has a high change impact on other system elements; therefore changes to this component should be minimized so as to avoid propagating changes to the other system components. The combined risk method can be used for the change propagation investigation. For every component, a prioritised list of all affected components can be prepared based on the DSM direct connectivity. For example, Figure 1(5a) shows such a prioritised change risk list for Supplier (8). From the list, it can be seen that Quality Controller (18) and Kitchen units are supplied from manufacturer (3) and Appliances (9) are at highest risk if the Supplier (8) changes. However, the links to the components in the middle range of the risk values are not always obvious because these components are usually only indirectly connected. Such a prioritised list can help avoid oversight of change impacts on those components. Figure 1-5(c) details the links between Supplier (8) and Kitchen Layout (15). This propagation path analysis provides the risk value of how change trigger affects the target. 

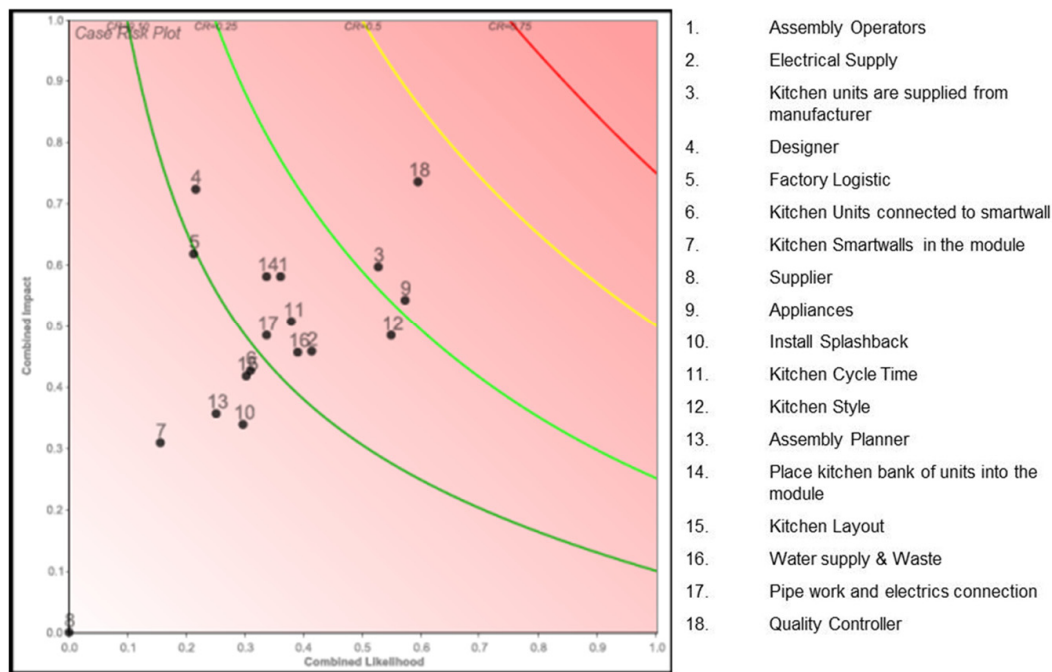

Figure 2. The combined risk plot with respect to the suppliers (in case-study)

In summary, Table 2 lists the highest risk elements by domain for the case study and shows that the Process domain has more high-risk elements than the other domains.

Table 2. The list of highest risk elements in the redesigned Kitchen Assembly System

\begin{tabular}{lllll}
\hline System Domains & System Elements & $C R$ & $C L$ & $C I$ \\
\hline Requirements & Kitchen Style & 0.27 & 0.55 & 0.48 \\
Product & Appliances & 0.31 & 0.57 & 0.60 \\
Process & Kitchen units are pre-assembled in a module & 0.32 & 0.53 & 0.60 \\
Organisation & Quality Controller & 0.44 & 0.60 & 0.74 \\
\hline
\end{tabular}

\section{Conclusion}

This paper presented a multi-domain change propagation model to assess the management of changes in MSD, which can be used to develop a design strategy to support decision making in manufacturing systems. This integrated MDM and CPM model supports the case study; (1) captures all system domains and the direct element dependencies both within and across domains; and (2) characterizes each dependency in terms of its likelihood and impact and provides a mechanism to propagate the risk of change across the entire system. The CPM is used to examine the direct and indirect change propagation between kitchen assembly system domains and elements to establish how the technique can be applied to provide the change likelihood, impact, and risk for each domain elements. By referring to the change indices, the changeability of engineering systems can be examined systematically. A heavy-duty diesel engine is used as an example in this work.

The use of MDM-CPM has been proposed as an integrated method for capturing the structure of the kitchen assembly system process, while also enabling an objective assessment of change propagated by varying elements of the system. This paper presented an empirical work to explore the potential benefits and limitations of integrated modelling. The integrated model requires additional validation using different empirical studies. While the method shows promise as indicated in the Kitchen Assembly System case study, we recognise that more work remains to be done. Future efforts should address the following research areas: (1) Understanding and test the usefulness of the method and the design 
strategies; the company's staff will subsequently examine the work. (2) The dependency between system elements and the requirement change relationships within manufacturing systems needs more efforts.

\section{Acknowledgement}

The authors would like to gratefully acknowledge Dr Tariq Masood and Seena Nair (from the Cambridge EDC), and Adam Robinson (from Laing O'Rourke) for their assistance on the case study.

\section{References}

Ahmad, N., Wynn, D.C. and Clarkson, P.J. (2013), "Change impact on a product and its redesign process: a tool for knowledge capture and reuse", Research in Engineering Design, Vol. 24 No. 3, pp. 219-244. https://doi.org/10.1007/s00163-012-0139-8

Ariyo, O.O., Keller, R., Eckert, C.M. and Clarkson, P.J. (2007), "Predicting Change Propagation on Different Levels of Granularity: An Algorithmic View", Proceedings of the ICED'07 / $16^{\text {th }}$ International Conference on Engineering Design, Paris, France, August 28-31, 2007, The Design Society, Glasgow, pp. 655-656.

Browning, T.R. (2001), "Applying the Design Structure Matrix to System Decomposition and Integration Problems: a Review and New Directions”, IEEE Transactions on Engineering Management, Vol. 48 No. 3 , pp. 292-306.

Clarkson, P.J., Simons, C. and Eckert, C. (2004), "Predicting Change Propagation in Complex Design”, Journal of Mechanical Design, Vol. 126 No. 5, pp. 788-797. https://doi.org/10.1115/1.1765117

Cochran, D.S., Arinez, J.F., Duda, J.W. and Linck, J. (2001), “A decomposition approach for manufacturing system design”, Journal of Manufacturing Systems, Vol. 20 No. 6, pp. 371-389. https://doi.org/10.1016/S02786125(01)80058-3

Eckert, C., Clarkson, P. and Zanker, W. (2004), "Change and Customization in Complex Engineering Domains", Research in Engineering Design, Vol. 15 No. 1, pp. 1-21. https://doi.org/10.1007/s00163-003-0031-7

Eger, T., Eckert, C.M. and Clarkson, P.J. (2005), "The Role of Design Freeze in Product Development", Proceedings of the ICED'05 / $15^{\text {th }}$ International Conference on Engineering Design, Melburne, Australia.

Eichinger, M., Maurer, M. and Lindemann, U. (2006), "Using Multiple Design Structure Matrices", Proceedings of the DESING $2006 / 9^{\text {th }}$ International Design Conference, Dubrovnik, Croatia, pp. 229-236.

Eppinger, S.D and Browning, T.R. (2012), Design Structure Matrix Methods and Applications, MIT Press.

Furtmeier, F. and Tommelein, I.D. (2010), "Explorative Application of the Multi-Domain Matrix Methodology in Lean Design", Proceedings of the 18th Annual Conference of the International Group for Lean Construction, Haifa, Israel, pp. 151-160.

Giffin, M., de Weck, O., Bounova, G., Keller, R., Eckert, C. and Clarkson, P.J. (2009), “Change Propagation Analysis in Complex Technical Systems”, Journal of Mechanical Design, Vol. 131 No. 8, pp. 081001. https://doi.org/10.1115/1.3149847

Hamraz, B., Caldwell, N.H.M. and Clarkson, P.J. (2013), “A holistic categorisation framework for literature on engineering change management", Systems Engineering, Vol. 16 No. 4, pp. 473-505. https://doi.org/10.1002/sys.21244

Hassannezhad, M., Cassidy, S. and Clarkson, P.J. (2017), "Dynamic Modelling of Relationships in Complex Service Design Systems", Proceedings of the ICED17 / $21^{\text {st }}$ International Conference on Engineering Design, Vol. 2, Vancouver, Canada, August 21-25, 2017, The Design Society, Glasgow, pp. 219-228.

Kim, Y.S. (2004), “A Decomposition Based Approach to Integrate Product Design and Manufacturing System Design”, Proceedings of the ICAD 2004 / $3^{\text {rd }}$ International Conference on Axiomatic Design, Seoul, June 2124, 2004.

Koh, E.C.Y., Caldwell, N.H.M. and Clarkson, P.J. (2012), "A method to assess the effects of engineering change propagation”, Research in Engineering Design, Vol. 23 No. 4, pp. 329-351. https://doi.org/10.1007/s00163012-0131-3

Koh, E.C.Y., Caldwell, N.H.M. and Clarkson, P.J. (2013), “A technique to assess the changeability of complex engineering systems", Journal of Engineering Design, Vol. 24 No. 7, pp. 477-498. https://doi.org/10.1080/09544828.2013.769207

Masmoudi, M., Leclaire, P., Zolghadri, M. and Haddar, M. (2017), "Change propagation prediction: A formal model for two-dimensional geometrical models of products", Concurrent Engineering: Research and Applications, Vol. 25 No. 2, pp. 174-189. https://doi.org/10.1177/1063293X17698192

Myklebust, O. (2002), Enterprise Modelling supported by Manufacturing Systems Theory, PhD Thesis, Norwegian University of Science and Technology, Trondheim, p. 19. 
Nylund, H., Salminen, K. and Andersson, P.H. (2009), “An approach to the integrated Design and Development of Manufacturing Systems", Proceedings of the 19th CIRP Design Conference, Cranfield University Press, pp. 448.

Pasqual, M.C. and de Weck, O.L. (2011), "Multilayer network model for analysis and management of change propagation", Proceedings of ICED'11 / $18^{\text {th }}$ International Conference on Engineering Design, Copenhagen, Denmark, August 15-18, 2011, pp. 126-138.

Reddi, K.R. and Moon, Y.B. (2009), "A Framework for Managing Engineering Change Propagation", International Journal of Innovation and Learning, Vol. 6 No. 5, pp. 461-476. https://doi.org/10.1504/IJIL.2009.02506

Vasantha, G.V.A., Roy, R., Lelah, A. and Brissaud, D. (2012), “A review of product-service systems design methodologies", Journal of Engineering Design, Vol. 23 No. 9, pp. 635-659. https://doi.org/10.1080/09544828.2011.639712

Vaughn, A., Fernandes, P. and Shields, J.T. (2002), Manufacturing System Design Framework Manual, MIT.

Wickel, M.C. and Lindemann, U. (2015), "How to build up an Engineering Change dependency model based on past change data?", Proceedings of the DSM 2015 / $17^{\text {th }}$ International Dependency and Structure Modeling Conference, Fort Worth, USA, November 4-6, 2015, Carl Hanser Verlag, pp. 221-231.

Wiendahl, H.P. and Heger, C.L. (2005), "Justifying Changeability. A Methodical Approach to Achieving Cost Effectiveness", The International Journal for Manufacturing Science and Production, Vol. 6 No. 1-2, pp. 33 40. https://doi.org/10.1515/IJMSP.2004.6.1-2.33

Wright, I.C. (1997), "A review of research into engineering change management: implications for product design", Design Studies, Vol. 18 No. 1, pp. 33-42. https://doi.org/10.1016/S0142-694X(96)00029-4

Wynn, D., Cassidy, S. and Clarkson, P.J. (2012), "Design of robust service operations using cybernetic principles and simulation", Proceedings of the DESIGN2012 / $12^{\text {th }}$ International Conference on Design, Dubrovnik, Croatia, The Design Society, pp. 331-342.

Hatice Olmez, PhD Student

University of Cambridge, University of Cambridge

3 Telford Close, E17 8SW London, United Kingdom

Email: ho277@eng.cam.ac.uk 